\title{
Article
}

\section{Hydrogel and Organogel Formation by Hierarchical Self-Assembly of Cyclic Peptides Nanotubes}

Shaikh, Huda, Lunn, Andrew, peltier, Raoul, Perrier, Sebastien, macdougall, Laura, Gurnani, Pratik, Yang, Jie, Huband, Steve and Mansfield, Ed

Available at https://clok.uclan.ac.uk/28418/

Shaikh, Huda, Lunn, Andrew orcid iconORCID: 0000-0003-2884-2755, peltier, Raoul, Perrier, Sebastien, macdougall, Laura, Gurnani, Pratik, Yang, Jie, Huband, Steve and Mansfield, Ed (2018) Hydrogel and Organogel Formation by Hierarchical Self-Assembly of Cyclic Peptides Nanotubes. Chemistry - A European Journal, 24 (71). pp. 19066-19074. ISSN 0947-6539

It is advisable to refer to the publisher's version if you intend to cite from the work. http://dx.doi.org/10.1002/chem.201804576

For more information about UCLan's research in this area go to

http://www.uclan.ac.uk/researchgroups/ and search for < name of research Group>.

For information about Research generally at UCLan please go to http://www.uclan.ac.uk/research/

All outputs in CLoK are protected by Intellectual Property Rights law, including Copyright law. Copyright, IPR and Moral Rights for the works on this site are retained by the individual authors and/or other copyright owners. Terms and conditions for use of this material are defined in the policies page.

\section{CLoK}

Central Lancashire online Knowledge www.clok.uclan.ac.uk

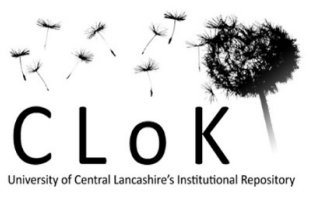




\title{
Hydrogel and Organogel Formation via Hierarchical Self- Assembly of Cyclic Peptides Nanotubes
}

\author{
H. Shaikh, ${ }^{[a]}$ J. Y. Rho, ${ }^{[a]}$ L. J. Macdougall, ${ }^{[a]}$ P. Gurnani, ${ }^{[a]}$ A. M. Lunn, ${ }^{[a]}$ J. Yang, ${ }^{[a]}$ S. Huband, ${ }^{[b]}$ E. D. H. \\ Mansfield, ${ }^{[a]}$ R. Peltier, ${ }^{*}{ }^{[a]}$ S. Perrier. ${ }^{[a, c, d]}$
}

\begin{abstract}
Breaking away from the linear structure of previouslyreported peptide-based gelators, this study reports the first example of gel formation based on the use of cyclic peptides made of alternating D- and L- amino acids, which self-assemble in solution to form long nanotubes. Herein, a library of cyclic peptides were systemically studied for their gelation properties in various solvents, uncovering key parameters driving both organogel and hydrogel formation. The hierarchical nature of the self-assembly process in water was characterised by a combination of electron microscopy imaging and Small Angle X-ray Scattering, revealing a porous network of entangled nanofibers composed by the aggregation of several cyclic peptide nanotubes. Rheology measurements then confirmed the formation of soft hydrogels with comparable stiffness to soft tissue.
\end{abstract}

\section{Introduction}

Hydrogels are fascinating materials that consist mostly of water molecules (typically $95 \%$ or above) being held together by networks of molecular fibres. Due to their high water content, these materials have become a popular choice in a wide range of applications such as contact lenses, wound dressing, injectable drug delivery systems or artificial matrixes for cell growth. ${ }^{[1],[2],[3]}$ While the majority of currently available hydrogels are made from chemically crosslinked synthetic polymer chains, peptides that self-assemble into physically cross-linked fibre-like structures have risen as a particularly promising alternative..$^{[4]}$ In addition to their inherent biocompatibility and biodegradability, these peptides rely on a convenient "bottom up" approach, in which a small change in the structure of the building block can induce dramatic changes in the physical property on the resulting materials. A variety of fibre-forming peptides have been

[a] Professor S. Perrier, Dr R. Peltier, Dr E. D. H. Mansfield, Ms H. Shaikh, Ms J. Y. Rho,, Ms L. J. Macdougall, Mr P. Gurnani, Mr A. M. Lunn, Mr J. Yang,

Department of Chemistry, University of Warwick, CV4 7AL, United Kingdom

E-mail: S.Perrier@warwick.ac.uk, R.Peltier@warwick.ac.uk

[b] Dr S. Huband

Department of Physics, University of Warwick, CV4 7AL, United Kingdom

[c] Professor S. Perrier

Warwick Medical School, University of Warwick, Coventry CV4 7AL, United Kingdom

[d] Professor S. Perrier

Faculty of Pharmacy and Pharmaceutical Sciences, Monash University, VIC 3052, Australia

Supporting information for this article is given via a link at the end of the document. developed recently. Examples include peptides that fibrillate upon formation of a bilayer $\beta$-sheet structure, ${ }^{[5]}$ collagen mimicking $\alpha$-helical peptides that self-assemble through coil-coil aggregation, ${ }^{[6]}$ and short $\mathrm{N}$-terminal protected phenylalanine-rich peptide sequence that stack via a combination of hydrophobic and $\pi-\pi$ stacking interactions.$^{[7]}$ Another advantage of using small molecule gelators is the possibility to prepare highly functional hydrogels by mixing functionalised building blocks in the gelating formulation. ${ }^{[8]}$

Over the years, examples of hydrogelating peptides have almost exclusively relied on linear structures. A range of cyclic tri- and dipeptides has been described previously as the smallest peptide-based hydrogelators..$^{[9]}$ Recently, Choi et al. demonstrated that cyclic peptides made out of 11 or 12 amino acids resulted in unusual self-assembly as compared to their linear equivalents. ${ }^{[10]}$ Recently, Méndez-Ardoy et al. reported on the formation of artificial fibrillar networks confined in water droplets using cyclic peptide nanotubes.(add ref.) Despite these promising results, the use of larger cyclic peptide structures as hydrogelating building blocks remains unexplored. In the 90's, Ghadiri and co-workers discovered that cyclic peptides (CPs) containing 8 to 12 amino acids of alternating $\mathrm{D}$ - and Lconfigurations are able to stack via $\beta$-sheet antiparallel $H$ bonding, leading to the formation of well-defined nanotubes with sizes ranging between 10 to $500 \mathrm{~nm}$, and cavity diameters between 2 to $13 \AA$ (Fig. 1). ${ }^{[11]}$ These systems offer an interesting versatility as the number of amino acids present in the peptide ring controls the internal diameter of the nanotubes whilst the nature of the amino acids and conjugated moieties change the properties of the external surface of the tubes. ${ }^{[12]}$ In addition, the planar structure of these peptides with the amino acid side chains protruding perpendicularly to the tubular axis offer a convenient handle for the functionalisation of the nanotubes without disrupting the assembly. ${ }^{[13]}$ This was exploited to developed cyclic peptide-based nanotubes functionalised with hydrophilic polymers that were shown to self-assemble even when decorated with polymeric chains many times their molecular weight (up to $20 \mathrm{kDa}$ ), thus forming a soft functional layer on the tube surface. ${ }^{[14]}$ Interestingly, these self-assembling systems were shown to be highly dynamics with cyclic peptides functionalised with various moieties (polymer, dyes) readily mixing to incorporate different functionalities within the same nanotubes. ${ }^{[15]}$

Herein, we explore the use of these alternating D- and L- cyclic peptides as building blocks for the formation of gel materials in a variety of solvents, particularly in water. A library of 8-membered CPs was screened for their gelating properties, uncovering the key structural parameters driving gel formation. Due to the charged nature of some of the residues, the influence of $\mathrm{pH}$ on hydrogelation was carefully tested. Selected samples of 
hydrogels were then studied via electron-microscopy and smallangle X-ray scattering (SAXS) to assess the underlying structure

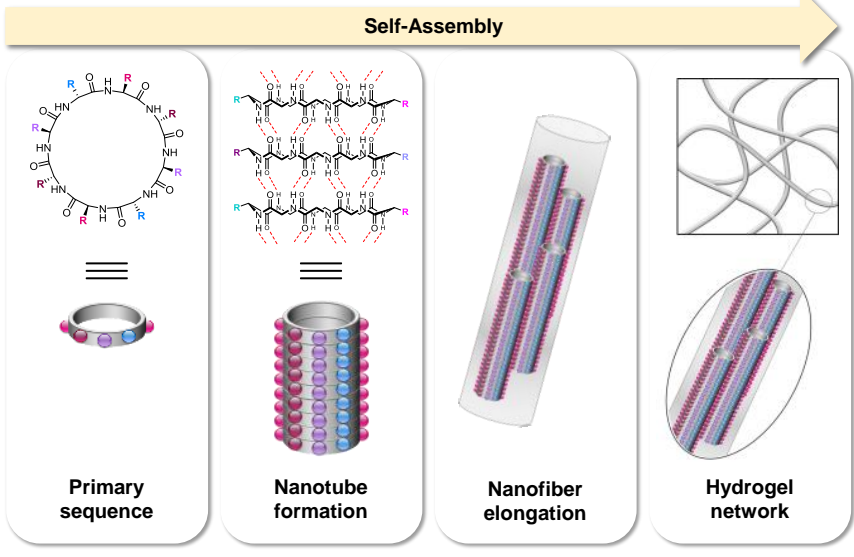

Figure 1. Hierarchical self-assembly process involved in hydrogel formation from D-, L- cyclic peptides.

of the hydrogels formed, and rheology measurements were used to characterise the physical properties of the materials.

\section{Results and Discussion}

Alternating D- and L-amino acid cyclic peptides (CPs) composed of eight $\alpha$-amino acids were considered for this study, as they are known to promote good self-assembly while being small enough to be easily synthesised. ${ }^{[16]}$ Peptides were designed to contain D-Leucine, as they have previously been shown to help promote the self-assembly process via increased hydrophobic interactions. ${ }^{[17]}$ These peptides also contains L-Tryptophan, which was empirically found by our group to improve peptide solubility in aqueous solutions. A library of CPs with varying Lresidues was then designed, in an iterative manner, starting from fully protected cyclic peptide $\mathbf{1}$ which was discovered to form a gel in DMF during previous studies by our group. The influence of protecting group on gelation was investigated using the product of selective deprotection of CP 1. In an attempt to balance the overall hydrophobicity of the structure, several hydrophilic residues (L-Lysine, L-Glutamic Acid or L-Cysteine) were then introduced in the structure, and the effect of varying the nature and number of these residues on gelation was carefully investigated.

Linear sequences were synthesised by solid phase peptide synthesis (SPPS) then cleaved from the resin using a mild acid (HFIP) to conserve the protecting groups. Cyclisation of the protected peptides was then achieved by the coupling of $N$ - to $C$-terminus using 4-(4,6-dimethoxy-1,3,5-triazin-2-yl)-4methylmorpholinium (DMTMM.BF 4 ) to form a stable amide bond, under dilute conditions to favour intramolecular cyclisation. Bocdeprotection was then carried out via treatment with TFA in the presence of scavengers. Cyclic peptides were purified via precipitation, either in a mixture of methanol and water (for the protected compounds), or in diethyl ether for the fully deprotected compounds. Cyclic peptides were characterised by electron spray ionisation-time-of-flight (ESI-TOF) mass spectroscopy (Table S1, ESI†) and their purity assessed using reverse-phase high-pressure liquid chromatography (RP-HPLC) (Fig. S2, ESI†). Cyclic peptides were found to have a purity superior to $85 \%$ and were used as such. A summary of the cyclic octapeptides used in this project is given in Scheme 1.

Cyclic peptide 1, which was initially discovered to form a transparent gel in DMF, was found to be fully soluble in DMSO and methanol (Table 1), with no gel formation. On the other hand, CP 1 did not dissolve in water, and remained as a white insoluble solid despite extensive sonication, due to the highly hydrophobic nature of the fully protected peptide. With the formation of a hydrogel in mind, we then decided to gradually decrease the hydrophobicity of CP 1 via selective removal of the orthogonal protecting groups (Scheme S1, ESI†). Hydrogelation or organogelation tests were carried out by dissolving the peptide powder directly in the indicated solvent, sonicating the mixture and letting it to rest at room temperature for 3 to 76 hours. Gelation was systematically tested via the vial inversion method (Table 1).

Selective deprotection of the Dde group using $4 \%$ hydrazine yielded CP 2 with Boc protecting groups still intact. CP 2 formed clear organogels in DMF, DMSO and methanol after approximately 3 hours, yet no gels could be formed in water. Selective deprotection of the Boc protecting groups from two Lysine and one Tryptophan residues, leaving one Dde-protected

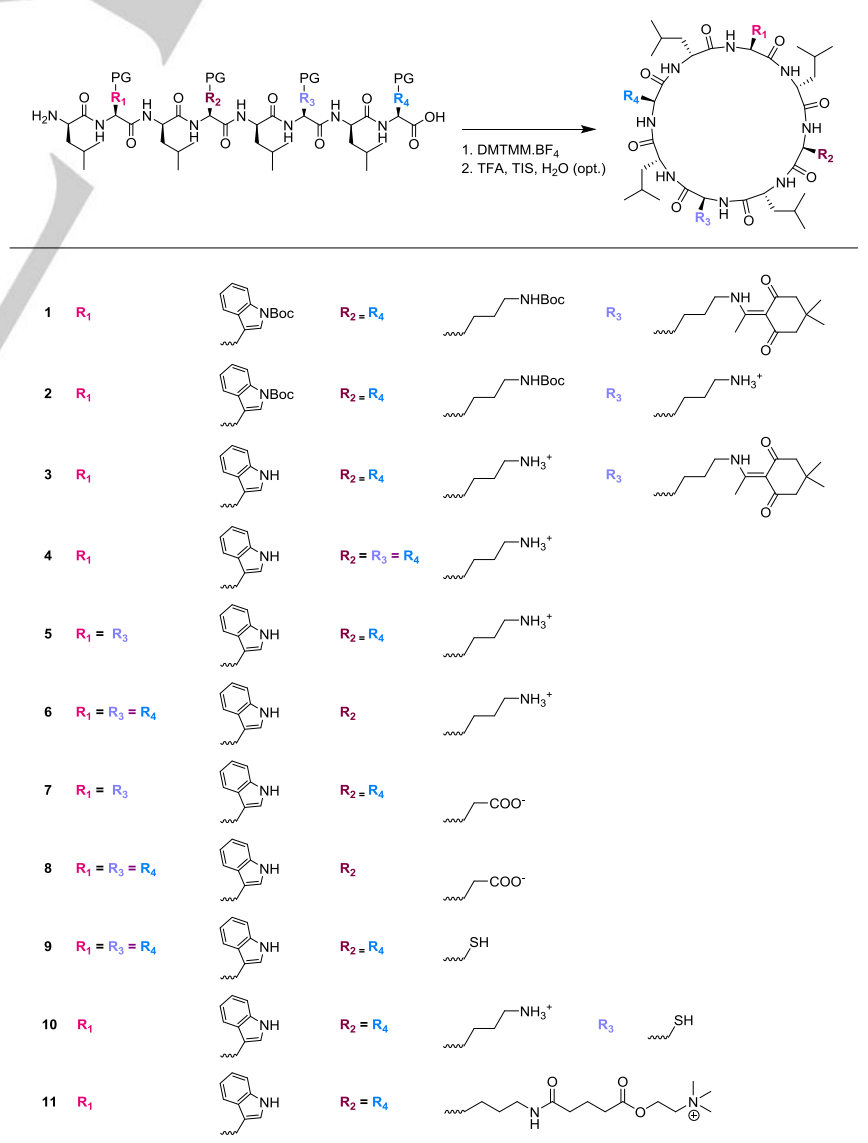

Lysine residue, yielded CP 3 which formed a gel in DMF and 
Table 1. Gelation properties of selected cyclic peptides in water and organic solvents. Hydrogelation assays were carried out at $\mathrm{pH}=3$ for peptides 1-6 \& 9-10 and $\mathrm{pH}=11$ for peptides $7-8$

\begin{tabular}{|c|c|c|c|c|c|c|c|c|c|c|c|c|}
\hline \multirow{2}{*}{ Solvent } & \multirow{2}{*}{ Concentration ${ }^{[a]}$} & \multicolumn{11}{|c|}{ Cyclic Peptide ${ }^{[b]}$} \\
\hline & & 1 & 2 & 3 & 4 & 5 & 6 & 7 & 8 & 9 & 10 & 11 \\
\hline \multirow[t]{3}{*}{ Water } & 5 & $\mathrm{P}$ & $\mathrm{P}$ & PS & S & G & $\mathrm{P}$ & $\mathbf{G}$ & $\mathrm{P}$ & $P$ & PS & G \\
\hline & 3 & $P$ & $P$ & PS & $S$ & G & $P$ & G & $P$ & $P$ & PS & G \\
\hline & 1 & $\mathrm{P}$ & $\mathrm{P}$ & PS & S & S & $\mathrm{P}$ & $S$ & $\mathrm{P}$ & P & PS & $\mathrm{S}$ \\
\hline DMSO & 2 & $\mathrm{~S}$ & G & $\mathrm{S}$ & G & $S$ & $\mathrm{~S}$ & G & G & - & - & - \\
\hline $\mathrm{MeOH}$ & 2 & $S$ & G & G & $S$ & $S$ & $S$ & G & G & - & - & - \\
\hline
\end{tabular}

[a] Concentration in w.t\% $[\mathrm{b}] \mathrm{G}=$ gel, $\mathrm{S}=$ solution, $\mathrm{PS}=$ partially soluble and $\mathrm{P}=$ precipitate

methanol. Peptide $\mathbf{3}$ was also found to be partially soluble in water $(\mathrm{pH}=3)$, but still not capable of forming a hydrogel. Finally, complete deprotection of $\mathbf{1}$ resulted in CP $\mathbf{4}$, which was fully soluble in water $(\mathrm{pH}=3)$ and methanol, but formed clear organogels in DMF and DMSO. No clear trend between organogel formation and polarity of the $\mathrm{CP}$ could be observed for CPs 1-4. Sequential removal of the hydrophobic protecting groups resulted in increased solubility in water as expected.

Despite covering a significant range of hydrophilicity with these compounds, none formed a hydrogel. To elucidate whether water-soluble CP 4 formed nanofibers in water nevertheless, TEM imaging of a 2 w.t \% sample of 4 (water, $\mathrm{pH}=3$ ), diluted 10 times to facilitate visualisation, was carried out. Images revealed a network of crosslinked fibres (Fig. 2A; Fig. S3, ESIt), indicating that self-assembly was taking place, however the constructs were not sufficiently dense to trap water molecules and form a hydrogel. The width distribution of the nanofibers, as measured from TEM images, indicates an average width of 30
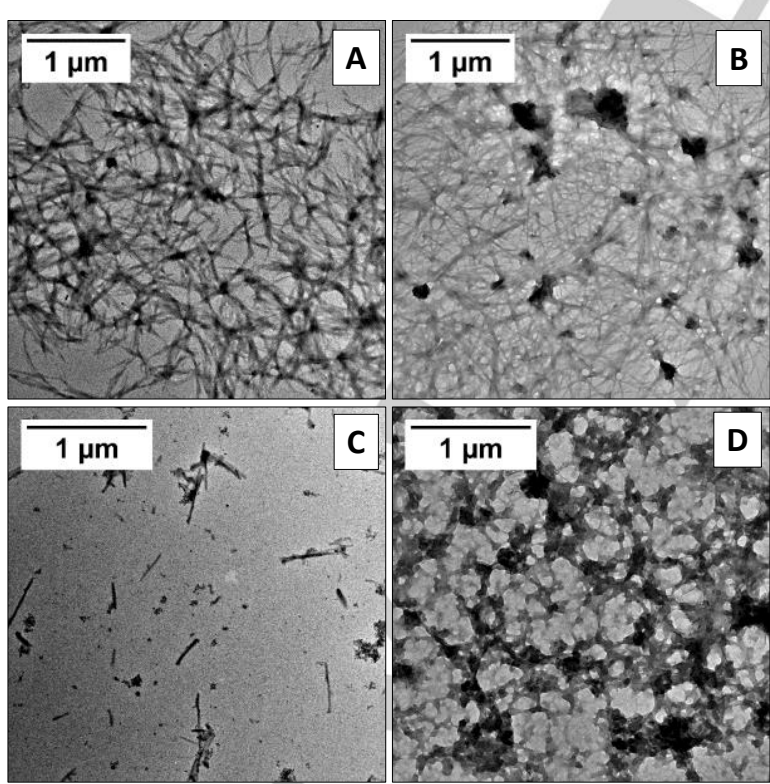

Figure 2. TEM images and fibre size distribution for a A) solution of $\mathbf{4}$ at $0.2 \mathrm{w} . \mathrm{t} \%$ in water $(\mathrm{pH}=3)$; B) solution of 5 at $0.3 \mathrm{w} . \mathrm{t} \%$ in water $(\mathrm{pH}=3)$ C) solution of 5 at 0.05 w.t \% in water $(\mathrm{pH}=3)$; D) solution of 7 at 0.4 w.t \% in water $(\mathrm{pH}=11)$. $\mathrm{nm}$. Given that single cyclic octapeptide nanotubes, having a diameter of approximatively $1 \mathrm{~nm},{ }^{[18]}$ this demonstrates that the fibres are composed of hundreds of laterally aggregated nanotubes that have formed nanotubular bundles. This is in accordance with previous work reporting the lateral aggregation of cyclic peptide-based nanotubes in aqueous solution. ${ }^{[19]}$ While the length of these fibers is difficult to judge, the fibril structure obtained here resembles the structure obtained for previouslyreported hydrogelating system. ${ }^{[20]}$

The formation of fibers from CP 4 suggests that hydrogel formation may be possible from this Lysine-functionalized cyclic peptide system, but that a fine balance between hydrophobicity and hydrophilicity needs to be achieved to enable formation of longer fibrils which can entangle into a denser network in aqueous environment. The influence of the number of charged residues was then investigated by varying the number of Lysine residues in CP 4 and substituting them with neutral Tryptophan residues instead. CP 5, containing a symmetrical arrangement of two Lysine and two Tryptophan residues on opposing sides, was found to form a clear organogel in DMF after 2 hours but was fully soluble in DMSO and methanol. Most interestingly, solutions of CP 5 in water $(\mathrm{pH}=3)$ at $3 \mathrm{wt} \%$ led to the formation of an opaque hydrogel after letting it rest for 16-24 hours at room temperature (Fig. S4A, ESI†). Decreasing the concentration to 1 wt $\%$ resulted in a slightly opaque solution which did not form a gel, nor formed a sediment at the bottom of the vial over time. SEM was employed to visualise the macrostructure of the hydrogel material formed. SEM imaging of a $3 \mathrm{wt} \%$ sample of CP $5(\mathrm{pH}=3)$ showed a porous network of elongated fibres, as typically observed for peptide-based hydrogels (Fig. S5). ${ }^{[21]}$ TEM imaging was then employed to visualise individual nanofibers with higher resolution. TEM images of a $0.3 \mathrm{w} . \mathrm{t} \%$ sample of CP $5(\mathrm{pH}=3)$, showed a network of entangled fibres with an average nanofiber width of $20 \mathrm{~nm}$ (Fig. 2A; Fig. S6, ESIt). The effect of dilution on fibre morphology was tested by imaging at a further 2 fold and 6 fold dilution (final concentration $=0.15$ and 0.05 w.t \%, Fig. 2C, Fig. S6, ESI†). Images showed nanofibers of comparable width but with decreasing length and density as concentration decreased. This is consistent with the absence of hydrogel formation at $1 \mathrm{wt} \%$ for CP $\mathbf{5}$, indicating that reducing the concentration does not form a sufficient fibril network to entrap water molecules and therefore form a hydrogel. 
Reducing the number of Lysine residues further, (mono-Lysine, CP 6) resulted in a compound that formed a white precipitate in water. In contrast with the opaque solution obtained with CP $\mathbf{5}$ at $1 \mathrm{wt} \%$, the present opaque solution settled at the bottom of the vial after a few hours. As expected, decreasing the number of charged residues resulted in increased hydrophobicity of the peptides (see HPLC in Fig. S2, ESI†) which translates into a reduction of solubility in water from a fully soluble compound to a hydrogel, then to a fully insoluble peptide. CP 6 was however fully soluble in DMF, DMSO and methanol. Fully deprotected CPs 4-6 show a trend in which increasing the number of charged residues, and thus hydrophilicity, favours gelation in organic solvents.

Having determined that two hydrophilic residues is the optimal combination for hydrogelation, we investigated the effect of modifying these residues. Firstly, the positively charged L-Lysine residues in CP $\mathbf{5}$ and $\mathbf{6}$ were replaced by negatively charged LGlutamic acid residues (CPs 7 and 8). CP 8 was found to form a gel in DMSO and methanol, while CP 7 formed a gel in all three organic solvents tested. Overall, these results indicate that the nature of the charge is not essential in forming organogels, and that increasing the number of charged residues in the CPs appear to favour gelation in organic solvent, possibly due to a reduction of the solubility of the peptides in these solvents.

In water at $\mathrm{pH} 11$, mono-Glutamic acid $\mathrm{CP} 8$ precipitated in solution, while hydrogels were obtained for di-Glutamic acid CP 7 at concentrations above 3 w.t \%, following 4-6 hours incubation at room temperature (Fig. S4B, ESIT). A comparison of the fibres obtained for CP 7 and 5 via TEM imaging shows that while both form a porous macroscopic network of fibres is formed, di-Glutamic peptide 7 forms nanofibers have wider widths and potentially shorter lengths than its di-Lysine counterpart CP 5 (Fig. 2D, Fig. S6, ESI†). While the mechanism of self-assembly is expected to be the same for positively and negatively charged compounds, a number of factors are expected to affect the solubility and hydrophilicity of the final compounds, and thus account for these differences. These include the nature of the counterions, the purity of the final peptides, or difficulties in accurately tuning the $\mathrm{pH}$ due to the small volumes tested.

Replacement of the charged residues by uncharged hydrophilic residues, such as Cysteine in CP 9, resulted in a peptide that is not soluble in water, further highlighting the importance of charges in hydrogel formation. It is noteworthy that similar results were obtained in the presence or absence of TCEP as a reducing agent to avoid lateral crosslinking via disulphide bond formation.

As expected due to the presence of charged residues ( $\mathrm{pKa}$ Lysine $=10.65, \mathrm{pKa}_{\text {Glutamic Acid }}=4.15$ ), hydrogels from CPs 5 and 7 exhibited a clear dependence on $\mathrm{pH}$. This behaviour was carefully characterised for the compounds containing charged residues (4-8 and 10). The results, reported in Table 2, show that $\mathrm{CP} \mathbf{5}$ forms a hydrogel exclusively between $\mathrm{pH}=2$ and $\mathrm{pH}$ $=4$. At $\mathrm{pH}>4$, partial protonation of these residues resulted in the peptide becoming partially insoluble, while at $\mathrm{pH}<2$ the increased number protonated Lysine resulted in a fully soluble compound instead. For similar reasons, CP 7 formed a hydrogel at $\mathrm{pH}>$ 9. Interestingly, Tri-Lysine CP 4 failed to form a hydrogel but went from an opaque solution to a precipitate at around $\mathrm{pH}=$ 5 , suggesting that the number of charged residues plays an important role in the gelation process.

The relationship between the presence of charges and gelation was further explored by preparing a $\mathrm{CP}$ bearing two ammonium moieties, which remains positively charged regardless of the $\mathrm{pH}$. CP 11 was prepared in one step by modifying CP 5 using glutaric acid choline ester. As expected, CP $\mathbf{1 1}$ formed a clear hydrogel at $3 \mathrm{wt} \%$ at $\mathrm{pH}=2, \mathrm{pH}=7$ and $\mathrm{pH}=12$ (Fig S4C), confirming that the presence of charge is an essential criterion to hydrogel formation for the studied compounds. Although, the charge exhibited by the lysine, glutamic acid or ammonium residues should result in electrostatic repulsion between individual cyclic peptides preventing self-assembly, the opposite is observed. We hypothesise that the stacking of these CPs partly occurs in such a such way that each peptide in the assembly is adopting an alternating conformation, where the charged residues $\left(R_{1}\right.$ and $R_{3}$ ) of one cyclic peptide aligns with the non-charged Tryptophan residues $\left(R_{2}\right.$ and $\left.R_{4}\right)$ of the subsequent peptide in the stack. However, the observation of a $2 \mathrm{~nm}$ red-shift of the UV spectra in the region corresponding to Tryptophan for aqueous solutions of $\mathbf{5}$ and $\mathbf{7}$, as compared to bulk Tryptophan in solution, suggests that at least some pi-pi stacking is involved in the assembly process. (ref)

Hence, the importance of Tryptophan residues on hydrogel formation was further assessed by replacing one hydrophobic Tryptophan residue from CP 5 , by a hydrophilic Cysteine residue to give $\mathrm{CP} \mathbf{1 0}$ instead. As expected, the solubility was dependent on $\mathrm{pH}$, with $\mathrm{CP} \mathbf{1 0}$ forming an opaque solution at $\mathrm{pH}$ $\leq 3$, but precipitating out of the solution at higher $\mathrm{pHs}$ without forming hydrogels. While such substitution increases the overall hydrophilicity of CP $\mathbf{1 0}$ as compared to CP $\mathbf{5}$ (See Fig. S2, ESI†), removal of a Tryptophan residues appears to diminish the peptide solubility in water. This is in accordance with general observations made in our group that Tryptophan residues increase the peptide solubility in aqueous solutions, arguably because they help reduce lateral aggregation of the individual nanotubes. ${ }^{[22]}$

The mechanical properties of the hydrogels produced by the dilysine 5, di-glutamic acid $\mathbf{7}$ and di-ammonium cyclic peptides $\mathbf{1 1}$

Table 2. Influence of $\mathrm{pH}$ on the gelation of selected cyclic peptides in water.

\begin{tabular}{cccccccccccccccc}
\hline $\begin{array}{c}\text { Cyclic } \\
\text { Peptide }\end{array}$ & 1 & 2 & 3 & 4 & 5 & 6 & 7 & 8 & 9 & 10 & 11 & 12 & 13 & 14 \\
\hline $\mathbf{4}$ & S & S & S & S & PS & PS & - & PS & - & PS & - & - & - & - \\
$\mathbf{5}$ & S & G & G & G & PS & PS & PS & - & - & - & - & - & - & - \\
$\mathbf{6}$ & P & - & P & - & P & - & P & - & - & - & - & - & - & - \\
$\mathbf{7}$ & P & - & P & - & P & - & P & PS & G & G & G & G & G & G \\
$\mathbf{8}$ & - & - & - & - & - & - & - & $P$ & - & P & - & P & - & P \\
$\mathbf{1 0}$ & PS & - & PS & - & P & - & P & - & P & - & - & - & - & - \\
$\mathbf{1 1}$ & - & G & - & - & - & - & G & - & - & - & - & G & - & - \\
\hline
\end{tabular}


were studied using rheological measurements. Oscillation frequency sweeps were carried out on hydrogels made from 5 (3 w.t $\%, \mathrm{pH}=3), 7$ (4 w.t\%, $\mathrm{pH}=10)$ and 11 (3 w.t \%, $\mathrm{pH}=7$ ). The experiment demonstrates the materials response to deformations and reveals the hydrogels viscoelastic properties. The samples exhibit a higher storage moduli $\left(G^{\prime}\right)$ than loss moduli (G') confirming the gel like state of the samples. The frequency-independent behaviour confirms the materials behave like an elastic solid at low frequency/ long timescales and at high frequency/ fast timescales (Fig. 3A, Fig S8A,C). ${ }^{[23]}$ The low absolute values measured for $G$ ' in the linear viscoelastic regime indicates that the gels formed are weak gels. Amplitude sweeps were carried out to monitor the response $G^{\prime}$ and $G$ " had with increasing strain (Fig. 3B, Fig S8B,D). The gel nature of the samples is confirmed by the $G$ ' values compared to the $G$ " values by one order of magnitude. The absence of a cross point of the G' and G" at high strain, as commonly observed amongst other supramolecular hydrogels, indicates that the hydrogel is stable up until $100 \%$ strain, which may be attributed to the highly dynamic nature of these self-assembled systems. ${ }^{[15]}$ This is in accordance with previous observations in the literature where similar systems showed a decrease in the elastic modulus only at strains higher than $100 \% .{ }^{[24],[25]}$ Taken together, the results suggest that, despite minor differences in the storage modulus of hydrogels made from CP 5, CP 7 and CP 11, all three hydrogels show very similar rheological behaviour.

Finally, SAXS experiments were carried out to discern more details about the structure of the individual fibers forming the hydrogel. Raw scattering data obtained for gel samples from 5 ( 3 w.t \%, pH = 3) and 7 (4 w.t \%, pH = 10) show significant

Figure 3. Rheology of cyclic peptide hydrogel 5. A) Frequency sweep of a hydrogel of $5(3$ w.t \%, water, $\mathrm{pH}=3)$ carried out at a temperature of $22{ }^{\circ} \mathrm{C}$ and $0.5 \%$ strain. B) Amplitude sweep of a hydrogel of 5 (3 w.t \%, water, $\mathrm{pH}=$
3) carried out at a temperature of $22^{\circ} \mathrm{C}$ and a frequency of $10 \mathrm{rad} \mathrm{s}^{-1}$.

differences between the two gels, especially given the minor differences in chemical structure. The absence of turn over at low $Q$ values for both data sets suggests a fractal like scattering, which is consistent with the structure of entangled polymer gels observed by TEM. ${ }^{[26]}$ To determine information on the higher order structure within the system, data were fitted to a previously-reported combination of cylindrical model and mass fractal model (Fig. S7 and S8, ESIt). ${ }^{[27]}$ The choice of a cylindrical model is based upon previous data reported for similar cyclic peptides, ${ }^{[28]}$ while the mass fractal model should account for their entanglement.

Using this model, an accurate fit was decided upon when Chi2 < 2. A mass fractal model describes the power law scattering, where the mass $M$ is proportional to the radius $(R)$ as a function of the fractional dimension $D f\left(M \sim R^{D f}\right)$. The fractal dimension is a statistical determination on the complexity of the gel network, with $D f=1$ for systems that are loosely aggregated with an illdefined structure and $D f=3$ for more densely aggregated structures. The correlation length represents the length of a single fiber, and the fractal radius refers to the radius of the fibers. This additive model takes into account the scattering from cylindrical-like aggregates that forms the basis of the gel structure. In both cases, the correlation length was fixed at 2000 $\AA$ since the maximum length of the fibers was found to be outside the window of observation for the SAXS instrument used in these investigations (2000 $\AA$ ), as evidenced by the TEM/SEM

Figure 3. SAXS scattering data obtained for solution of 5 ( 3 w.t \%, water, $\mathrm{pH}=$ 3) and 7 (4 w.t \%, water, $\mathrm{pH}=10)$. A) Scattering plot with drawn lines represent the associated power laws modelled to each sample (see SI for details). B) Summary of parameters used for fitting to a flexible cylinder model.
A

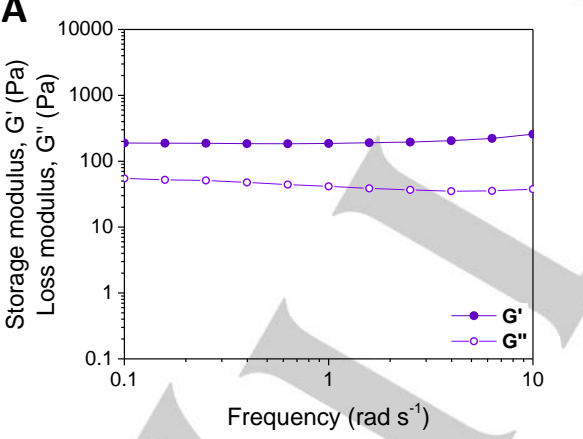

B

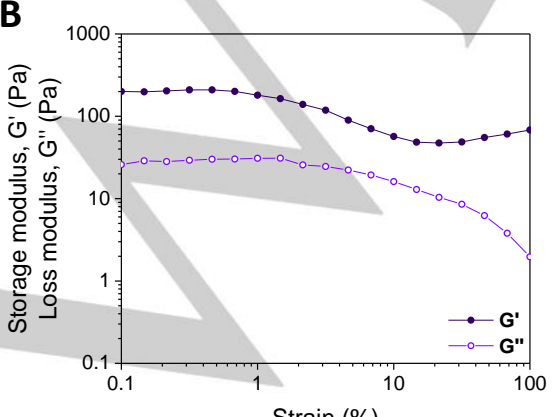

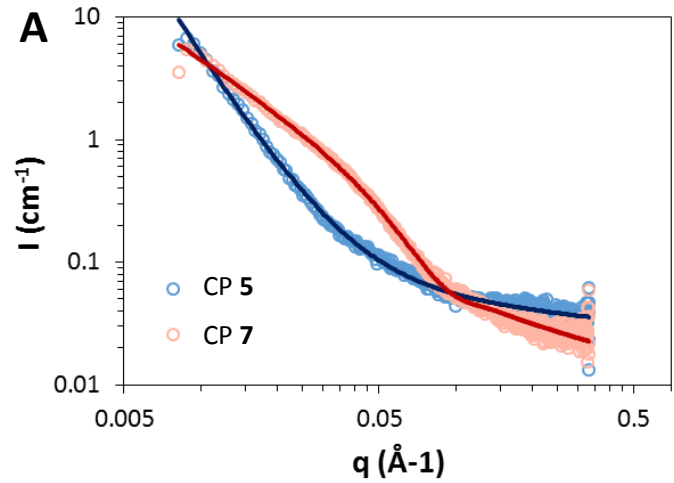

B

\begin{tabular}{|c|c|c|}
\hline Sample & $\begin{array}{c}\text { CP } 5 \\
(3 \mathrm{wt} \%)\end{array}$ & $\begin{array}{c}\text { CP } 7 \\
(4 \text { wt } \%)\end{array}$ \\
\hline Cylinder Radius (Å) & 5 & 5 \\
\hline Cylinder Length (Å) & 2000 & 418 \\
\hline Fractal Radius (Å) & 39 & 21 \\
\hline Correlation Length (Å) & 2000 & 2000 \\
\hline Fractal Dimension & 2.9 & 1.5 \\
\hline Chi2 & 1.5 & 1.7 \\
\hline
\end{tabular}

imag es. Addi tiona lly, the radiu $s$ of the cylin der was fixed at 5 Å. Whil e the widt $h$ of a singl e 
cyclic peptide nanotube varies depending on the type of technique used

to determine it, ${ }^{[19,29]}$ a diameter of $1 \mathrm{~nm}$ has previously been shown by SAXS and SANS to provide an optimal fit for the data. ${ }^{[30]}$ The fits obtained for CP $\mathbf{5}$ scattering data suggest the formation of long thin fibers with long length scales and a radius of approximately $40 \AA$. The fractal dimension obtained (2.9) suggests a well ordered and strongly aggregating system. This is in accordance with previously reported observation for similar polymeric hydrogels. ${ }^{[31]}$ In the case of $\mathrm{CP} 7$, long fibers are also formed, with lengths superior to $2000 \AA$. However, the individual cyclic peptide chains appear to be shorter $(418 \AA)$. The fractal dimension of 1.5 obtained from the fit suggests a weaker aggregation with a less ordered structure. While this tends to disagree with the similar mechanical behavior observed via rheology for these two systems, it matches the difference observed via TEM imaging, where shorter fiber lengths were shown in the case of $\mathrm{CP} 7(0.4 \mathrm{wt} \%, \mathrm{pH}=10)$ in comparison with $\mathrm{CP} 5(0.3 \mathrm{wt} \%, \mathrm{pH}=3)$.

\section{Conclusions}

A new class of gelators, based on a cyclic peptide motif, has been successfully identified via a careful study on the effect of structure and environmental conditions on the gelation of these compounds. Hydrogels that are responsive to $\mathrm{pH}$ have been formed from cyclic peptides containing two charged residues, either Lysine or Glutamic acid residues, while $\mathrm{pH}$-independent hydrogels could be formed using quaternized functional groups. These hydrogels were shown to occur via a hierarchical selfassembly process in which cyclic peptides first stack vertically into hollow nanotubes; these nanotubes then aggregate into larger fibres which tangle into a dense network that can entrap solvent molecules. To our knowledge, this is the first report of hydrogels formed from cyclic peptide nanotubes.

\section{Experimental Section}

\section{Materials}

All Fmoc protected amino acids and 2-(6-Chloro-1-H-benzotriazole-1-yl)1,1,3,3-tetramethylaminium hexafluorophosphate (HCTU) were used as received from Iris Biotech GMBW. Piperidine was purchased from Honeywell, (N, N- Diisopropylethylamine), (DIEA) from Fluka Analytical, trifluoroacetic acid (TFA) from Fluorochem and were all used as received 4-(4,6-dimethoxy-1,3,5-triazin-2- $\quad y l)-4$-methylmorpholinium tetrafluoroborate reagent $\left(\mathrm{DMTMM} \mathrm{BF}_{4}\right)$ was prepared as per literature procedure.[32]

\section{Synthesis of cyclic peptides 1-10}

Fully protected linear octapeptides were prepared via solid phase peptide synthesis (SPPS) on a Prelude Automated Peptide Synthesizer ${ }^{\mathrm{TM}}$ (Protein Technologies Inc.) using 2-chlorotrityl chloride resin as the solid support. The first Fmoc-protected amino acid was coupled to the resin using DIPEA (4 eq.) in DCM, followed by capping of unreacted resin sites using a solution of MeOH:DIPEA:DCM (7:1:2, v/v/v). Deprotection of the
Fmoc- group of the amino acids was done using $20 \%$ piperidine in DMF. Subsequent amino acids were coupled using Fmoc-amino acid (5 eq.), HCTU (5 eq.) and NMM (10 eq.) in DMF. In the last step, the linear octapeptides were cleaved from the resin (while keeping protecting groups on) by a solution of 20 vol \% 1,1,1,3,3,3-hexafluoro-2-propanol (HFIP) in DCM. Peptides were cyclised by stirring at room temperature for 3 to 5 days in the presence 1.2 equivalents of DMTMM. BF 4 in DMF. The solution was then concentrated in vacuo and precipitated with an ice-cold solution of $\mathrm{MeOH}$ : $\mathrm{H} 2 \mathrm{O}$ (1:1). Removal of the -Boc and -tBu protecting groups was achieved by adding a mixture of trifluoroacetic acid:triisopropylsilane:water (95:2.5:2.5 v/v/v) to the cyclic peptide and stirring for 3 to 4 hours. The resulting solution was then concentrated and precipitated in ice cold diethyl ether to give the fully deprotected cyclic peptides. -Dde deprotection was achieved via addition of a $4 \%$ hydrazine solution in DMF to the cyclic peptide and stirring for 3 hours. Precipitation in a 1:1 methanol:water and repeated washings in ice cold diethyl ether yielded the -Dde deprotected product. Peptides were dissolved or suspended in water and freeze dried to obtain a white powder. When necessary, cyclic peptides were purified via semipreparative RP-HPLC. Final peptides were characterized by mass spectrometry (electrospray ionization, ESI) (Table S1). The purity of the most hydrophilic ones was assessed using RP-HPLC (Figure S1).

\section{Cyclic Peptide 11}

Cyclic peptide $5(100 \mathrm{mg}, 0.092 \mathrm{mmol})$, hydrogen choline chloride glutarate $(35 \mathrm{mg}, 1.5$ eq., $0.14 \mathrm{mmol})$ and HBTU (68 mg, 2 eq., 0.18 $\mathrm{mmol})$ were solubilized into $10 \mathrm{~mL}$ anhydrous DMSO. NMM ( $28 \mathrm{mg}, 3$ eq., $0.28 \mathrm{mmol}$ ) was added and the reaction mixture was left to stir at room temperature for 3 hours. After the reaction, DMSO was removed using a stream of $\mathrm{N}_{2}$ and the mixture was dissolved in $\mathrm{CH}_{3} \mathrm{CN}$ and purified from the excess monomer and HBTU using a dialysis bag with a molecular weight cut off of $1 \mathrm{kDa}$. Tetrabutylammonium bromide was then added into the isolated cyclic peptide, and the precipitation was collected by centrifugation and dried in vacuum oven. Yield $=110 \mathrm{mg}(80 \%) .{ }^{1} \mathrm{H}$ NMR (400 MHz, TFA- $d, \mathrm{ppm}): \delta=8.01-7.35$ (m, 10H, Trp) , $5.77\left(\mathrm{~m}, 2 \mathrm{H}, \mathrm{H}_{\alpha}\right.$ Trp), 5.53 (m, 6H peptide, $\mathrm{H}_{\alpha}$ Leu and $\left.\mathrm{H}_{\alpha} \mathrm{Lys}\right), 5.01$ (m, 4H, COO- $\mathrm{CH}_{2}$ ), $4.08\left(\mathrm{~m}, 4 \mathrm{H}, \mathrm{CH}_{2}-\mathrm{N}\left(\mathrm{CH}_{3}\right) 3\right), 3.79\left(\mathrm{~m}, 4 \mathrm{H}, \mathrm{CH}_{2}-\mathrm{NH}-\mathrm{CO}\right), 3.57(\mathrm{~s}, 18 \mathrm{H}$, $\left.\mathrm{N}\left(\mathrm{CH}_{3}\right) 3\right), 3.13\left(\mathrm{~m}, 4 \mathrm{H}, \mathrm{NH}-\mathrm{CO}-\mathrm{CH}_{2}\right), 2.99\left(\mathrm{~m}, 4 \mathrm{H}, \mathrm{CH}_{2}-\mathrm{COO}\right), 2.44(\mathrm{~m}$, $\left.4 \mathrm{H}, \mathrm{NH}-\mathrm{CO}-\mathrm{CH}_{2}-\mathrm{CH}_{2}\right), 2.26-1.78\left(\mathrm{~m}, 12 \mathrm{H}, \mathrm{CH}_{2}-\mathrm{CH}_{2}-\mathrm{CH}_{2} \mathrm{Lys}\right), 1.81-1.40$ (m, 12H, CH${ }_{2}-\mathrm{CH}$ Leu), 1.30-1.06 (m, 24H, CH $\mathrm{CH}_{3}$ Leu). MS: [M-Br] ${ }^{+}$ calculated: 1561.8 , found: 1561.8 .

\section{Preparation of cyclic peptide gels}

Samples for gelation assay. Peptide powders were first weighted in 1.5 $\mathrm{mL}$ glass vials. Distilled water, dimethylformamide, dimethyl sulfoxide or methanol were then directly added onto the peptide powder to reach the desired concentration. Samples containing deprotected Lysine residues in water were then adjusted to $\mathrm{pH}=3$ using small volumes of $1 \mathrm{M} \mathrm{HCl}$ (typically 1 to $2 \mathrm{uL}$ ), while samples containing deprotected Glutamic acid residues were adjusted to $\mathrm{pH}=11$ using small volumes of $1 \mathrm{M} \mathrm{NaOH}$ (typically 1 to $5 \mathrm{uL}$ ). Samples were vortexed for 2 minutes and sonicated for $1 \mathrm{hr}$ at $40^{\circ} \mathrm{C}$, before being allowed to rest at room temperature for 3 to 76 hours. To help induce hydrogel formation, different triggers for gelation were tested including physical and chemical stimuli such as altering the temperature, sonication period, lowering and increasing the $\mathrm{pH}$ and predisolving the compounds in a small volume of good solvent to help solubilise the peptide. However, results obtained with these methods were identical to the ones reported using the method reported hereabove (Table 1), and therefore were omitted from this report for clarity. 
Sample for $\mathbf{p H}$ investigation. Peptide powders were first weighted in $1.5 \mathrm{~mL}$ glass vials. Distilled water was added to the peptide powders to reach the desired concentrations. The sample was vortexed for 2 minutes and sonicated for 20 minutes. The $\mathrm{pH}$ was then adjusted to the desired values using small volumes of either $1 \mathrm{M} \mathrm{HCl}(1$ to $5 \mathrm{uL}$ ) or $1 \mathrm{M}$ $\mathrm{NaOH}$ (1 to $15 \mathrm{uL}$ ). The sample was then vortexed for 2 minutes and sonicated for $1 \mathrm{hr}$, then allowed to gelate at room temperature for 16 to 76 hours.

\section{Characterisation}

High performance liquid chromatography (HPLC). Chromatograms were obtained on an Agilent 1260 Infinity series stack equipped with an Agilent 1260 variable wavelength detector. For analytical, the HPLC was fitted with a Phenomenex Luna ${ }^{\circledR} \mathrm{C} 18(250 \times 4.6 \mathrm{~mm})$ with $5 \mu \mathrm{m}$ micron packing (100 $\AA$ ). For semi-preparative purification, the HPLC was fitted with a Phenomenex Luna $^{\circledR} \mathrm{C} 18(250 \times 4.6 \mathrm{~mm})$ with $5 \mu \mathrm{m}$ micron packing $(100 \AA)$. Mobile phase A consisted of water containing $0.05 \%$ TFA, mobile Phase $B$ consisted of methanol containing $0.05 \%$ TFA. The gradient used for HPLC analysis was increased from $5 \%$ to $95 \%$ B in 30 minutes. Detection was achieved via monitoring at $280 \mathrm{~nm}$ (Tryptophan absorbance). Purity was calculated using integration of the area under the curve using Agilent OpenLab ${ }^{\circledR}$ software

Mass spectrometry (ESI-TOF). Electrospray ionisation- time of fligh mass spectra of samples dissolved in pure methanol were recorded on a Bruker Micro-TOF using positive ionisation mode and a recording range from 500 to $1500 \mathrm{~m} / \mathrm{z}$.

Scanning electron microscopy (SEM). Cyclic peptide hydrogels were first prepared using the method described above. Small samples of gel were then either air-dried by leaving it open to air overnight, freeze-dried or vacuum-dried in a vacuum oven. Samples were then mounted on super smooth silicon chips and coated in gold for 15 seconds using a Polaron 5C 7640 sputter coater before being imaged using a Zeiss Supra 55VP Scanning Electron Microscope.

Transmission electron microscopy (TEM). Cyclic peptide hydrogels were first prepared using the method described above. Gel samples were diluted 10 to 60 -fold and a $10 \mu \mathrm{L}$ drop of the diluted sample was immediately deposited onto a hydrophobic surface (plastic petri dish). The carbon-coated surface of the TEM grid was then placed on the drop for 1 minute, then left to dry before imaging. A JEOL 2100FX Transmission Electron Microscope at an accelerating voltage of $200 \mathrm{kV}$ was used to carry out the imaging

Rheology. Cyclic peptide hydrogels were first prepared using the method described above. Samples were then pipetted onto the lower plate of the rheometer. Measurements were performed on an Anton Paar MCR 302 rheometer using a parallel plate with a diameter of $8 \mathrm{~mm}$ and measuring gap of $1 \mathrm{~mm}$. The normal force was kept constant at $0 \mathrm{~N}$ during measurements and all measurements were performed at $22^{\circ} \mathrm{C}$ (maintained by a Peltier system). When conducting the amplitude sweep, a constant frequency was applied of $10 \mathrm{rad}^{-1}$ and the strain was ramped logarithmically from $0.01 \%$ to $100 \%$. At each decade, six points were taken and these measurements were repeated in triplicate to calculate the average storage and loss moduli with increasing strain. For the frequency sweep, a constant strain of $0.5 \%$ was applied (predetermined to be within the linear viscoelastic regime) and the angular frequency was ramped logarithmically from $10 \mathrm{rad} . \mathrm{s}^{-1}$ to $0.1 \mathrm{rad} . \mathrm{s}^{-1}$. At each decade, 5 points were taken and these measurements were repeated in triplicate to calculate the average storage and loss moduli over the frequency range. All data was analysed using RheoCompass software.

Small Angle X-ray Scattering. Small-angle X-ray scattering (SAXS) measurements were made using a Xenocs Xeuss 2.0 equipped with a micro-focus $\mathrm{Cu} \mathrm{Ka}$ source collimated with Scatterless slits. The scattering was measured using a Pilatus $300 \mathrm{k}$ detector with a pixel size of $0.172 \mu \mathrm{m}$ $\times 0.172 \mu \mathrm{m}$. The distance between the detector and the sample was calibrated using silver behenate $\left(\mathrm{AgC}_{22} \mathrm{H}_{43} \mathrm{O}_{2}\right)$, giving a value of $1.199(5)$ $\mathrm{m}$. The magnitude of the scattering vector $(\mathrm{q})$ is given by $q=4 \pi \sin \theta / \lambda$, where $2 \theta$ is the angle between the incident and scattered $X$-rays and $\lambda$ is the wavelength of the incident $X$-rays. This gave a q range for the detector of $0.01 \AA^{-1}$ and $0.33 \AA^{-1}$. Samples were held between two Kapton windows with a sample thickness of $0.5 \mathrm{~mm}$. An azimuthal integration of the 2D scattering profile was performed and the resulting data corrected for the absorption, sample thickness and background from the sample holder. Finally, the scattering intensity was then rescaled to absolute intensity using glassy carbon as a standard. ${ }^{[33]}$

All data were modelled using the SASview programme, ${ }^{[34]}$ using predefined form factors. The best fit to the data was obtained from an additive model, combined of a cylinder and mass fractal form factor (Equations 1 and 2, respectively)

$$
P(q, \alpha)=\frac{\text { scale }}{V} F^{2}(q, \alpha) \cdot \sin (\alpha)+\text { background }
$$

Equation 1

\section{Where}

$$
F(q, \alpha)=2(\Delta \rho) V \frac{\sin \left(\frac{1}{2} q L \cos \alpha\right)}{\frac{1}{2} q L \cos \alpha} \frac{J_{1}(q R \sin \alpha)}{q R \sin \alpha}
$$

Here, $\alpha$ is the angle between the axis of the cylinder, and $V=\pi R^{2} L$ is the volume of the cylinder, $L$ is the length of the cylinder, $R$ is the radius, and $\Delta \rho$ is the scattering length density difference between the sample and solvent. $\mathrm{J} 1$ is the first order Bessel function

$$
\begin{gathered}
I(q)=\text { scale } \times P(q) S(q)+\text { background } \\
P(q)=F(q R)^{2} \\
F(x)=\frac{3[\sin (x)-x \cos (x)]}{x^{3}} \\
S(q)=\frac{\Gamma\left(D_{m}-1\right) \zeta^{D_{m-1}}}{\left[1+(q \zeta)^{2}\right]^{\left(D_{m}-1\right) / 2}} \frac{\left.\sin \left[D_{m}-1\right) \tan ^{-1}(q \zeta)\right]}{q} \\
\text { scale }=\text { scale factor } \times N V^{2}\left(\rho_{\text {particle }}-\rho_{\text {solvent }}\right)^{2}
\end{gathered}
$$

Equation 2

Where $R$ is the radius of the building block, $D_{m}$ is the mass fractal dimension, $\zeta$ is the cut-off length, $\rho_{\text {particle }}$ and $\rho_{\text {solvent }}$ is the scattering length density of the particle and solvent, respectively.

The combined form factor allows for the fitting of a mass fractal gel-like system consisting of cylindrical fibres. In all cases, the SLD values were calculated based on the atomic structure of the peptide and solvent, respectively, according to the following equation

$$
S L D=\frac{\sum_{i-1}^{n} Z_{R e}}{V_{m}}
$$

Where $V_{m}$ is the molecular volume, and $Z_{R e}$ is the atomic number if the $i^{\text {th }}$ atom, multiplied by the classical atomic radius of a single electron (2.81 $\times 10^{-13} \mathrm{~cm}$ ). Table $\mathrm{S} 2$ provides a breakdown of the fitting parameters obtained from the fit. Values marked * were fixed during the fitting 
process. A good fit was considered when $\mathrm{Chi}^{2}<2$. It should be noted that the maximum size of a scattering particle using the SAXS set-up described here was $2000 \AA$. As such, given no turn over was observed in the q-range and the data follows a power law function, the cut-off length was fixed at $2000 \AA$. Additionally, from previous studies (described in the main manuscript), the radius of the cylinder was fixed at $5 \AA$.

\section{Acknowledgements}

We thank the Royal Society Wolfson Merit Award (WM130055; SP), the Monash-Warwick Alliance (HS; JYR, PG, AML, SP) and the European Research Council (TUSUPO 647106; EDHM, RP, SP) for financial support.

Keywords: hydrogel $\bullet$ cyclic peptide $\cdot$ organogel $\bullet$ self-assembly - nanofibers

[1] E. Calo, V. V. Khutoryanskiy, Eur. Polym. J. 2015, 65, 252-267.

[2] aM. Zhou, A. M. Smith, A. K. Das, N. W. Hodson, R. F. Collins, R. V. Ulijn, J. E. Gough, Biomaterials 2009, 30, 2523-2530; bA. Altunbas, S. J. Lee, S. A. Rajasekaran, J. P. Schneider, D. J. Pochan, Biomaterials 2011, 32, 5906-5914.

[3] aA. C. Jen, M. C. Wake, A. G. Mikos, Biotechnol. Bioeng. 1996, 50, 357-364; bG. M. Sun, X. J. Zhang, Y. I. Shen, R. Sebastian, L. E. Dickinson, K. Fox-Talbot, M. Reinblatt, C. Steenbergen, J. W. Harmon, S. Gerecht, Proc. Natl. Acad. Sci. U. S. A. 2011, 108, 20976-20981.

[4] aW. Y. Seow, C. A. E. Hauser, Mater. Today 2014, 17, 381-388; bG. Fichman, E. Gazit, Acta Biomater. 2014, 10, 1671-1682.

[5] T. Y. Cheng, M. H. Chen, W. H. Chang, M. Y. Huang, T. W. Wang, Biomaterials 2013, 34, 2005-2016.

[6] E. F. Banwell, E. S. Abelardo, D. J. Adams, M. A. Birchall, A. Corrigan, A. M. Donald, M. Kirkland, L. C. Serpell, M. F. Butler, D. N. Woolfson, Nat. Mater. 2009, 8, 596-600.

[7] aA. M. Smith, R. J. Williams, C. Tang, P. Coppo, R. F. Collins, M. L. Turner, A. Saiani, R. V. Ulijn, Adv. Mater. 2008, 20, 37-41; bR. Orbach, L. Adler-Abramovich, S. Zigerson, I. Mironi-Harpaz, D. Seliktar, E. Gazit, Biomacromolecules 2009, 10, 2646-2651.

[8] al. R. Wheeldon, S. C. Barton, S. Banta, Biomacromolecules 2007, 8, 2990-2994; bZ. N. Mahmoud, S. B. Gunnoo, A. R. Thomson, J. M. Fletcher, D. N. Woolfson, Biomaterials 2011, 32, 3712-3720; cV. Jayawarna, S. M. Richardson, A. R. Hirst, N. W. Hodson, A. Saiani, J. E. Gough, R. V. Ulijn, Acta Biomater. 2009, 5, 934-943.

[9] aH. M. Geng, L. Ye, A. Y. Zhang, J. B. Li, Z. G. Feng, Langmuir 2016, 32, 4586-4594; bY. Ishihara, S. Kimura, Journal of Peptide Science 2010, 16, 110-114; cA. J. Kleinsmann, B. J. Nachtsheim, Chem. Commun. 2013, 49, 7818-7820; dS. Liu, A. M. Tang, M. L. Xie, Y. D. Zhao, J. Jiang, G. L. Liang, Angewandte Chemie-International Edition 2015, 54, 3639-3642.

[10] S. J. Choi, W. J. Jeong, S. K. Kang, M. Lee, E. Kim, D. Y. Ryu, Y. B. Lim, Biomacromolecules 2012, 13, 1991-1995.

[11] aM. R. Ghadiri, K. Kobayashi, J. R. Granja, R. K. Chadha, D. E. McRee, Angewandte Chemie International Edition In English 1995, 34, 93-95 bN. Khazanovich, J. R. Granja, D. E. McRee, R. a. Milligan, M. Ghadiri, JACS 1994, 116, 6011-6012; cJ. R. Granja, M. R. Ghadiri, JACS 1994, 116, 10785-10786.

[12] N. Rodriguez-Vazquez, M. Amorin, J. R. Granja, Org. Biomol. Chem. 2017, 15, 4490-4505

[13] R. J. Brea, C. Reiriz, J. R. Granja, Chem. Soc. Rev. 2010, 39, 14481456.
[14] aS. Catrouillet, J. C. Brendel, S. Larnaudie, T. Barlow, K. A. Jolliffe, S Perrier, Acs Macro Letters 2016, 5, 1119-1123; bM. Danial, C. M.-N Tran, P. G. Young, S. Perrier, K. A. Jolliffe, Nature Communications 2013, 4; cC. Julien, B. Markus, Small 2008, 4, 1008-1016; dC. Julien, S J. D. J. S., K. Alexey, S. Svetlana, B. Markus, Angew. Chem. Int. Ed. 2005, 44, 3297-3301; eM. G. J. Ten Cate, N. Severin, H. G. Börner Macromolecules 2006, 39, 7831-7838.

[15] J. Y. Rho, J. C. Brendel, L. R. MacFarlane, E. D. H. Mansfield, R. Peltier, S. Rogers, M. Hartlieb, S. Perrier, Adv. Func. Mater. 2017, 1704569.

[16] S. C. Larnaudie, J. C. Brendel, K. A. Jolliffe, S. Perrier, Journal of Polymer Science Part a-Polymer Chemistry 2016, 54, 1003-1011.

[17] R. Chapman, M. Danial, M. L. Koh, K. A. Jolliffe, S. Perrier, Chem. Soc. Rev. 2012, 41, 6023-6041.

[18] aM. Engels, D. Bashford, M. R. Ghadiri, JACS 1995, 117, 9151-9158; bM. R. Ghadiri, J. R. Granja, R. a. Milligan, D. E. McRee, N. Khazanovich, Nature 1993, 366, 324-327.

[19] M. R. Silk, J. Newman, J. C. Ratcliffe, J. F. White, T. Caradoc-Davies, J. R. Price, S. Perrier, P. E. Thompson, D. K. Chalmers, Chem. Commun. 2017, 53, 6613-6616.

[20] aR. Peltier, G. Chen, H. Lei, M. Zhang, L. Gao, S. S. Lee, Z. Wang, H. Sun, Chem. Commun. 2015, 51, 17273-17276; bK. Medini, B. W Mansel, M. A. K. Williams, M. A. Brimble, D. E. Williams, J. A. Gerrard Acta Biomater. 2016, 43, 30-37; cK. M. Galler, L. Aulisa, K. R. Regan, R. N. D'Souza, J. D. Hartgerink, JACS 2010, 132, 3217-3223.

[21] R. Huang, W. Qi, L. Feng, R. Su, Z. He, Soft Matter 2011, 7, 6222-6230.

[22] M. Danial, S. Perrier, K. A. Jolliffe, Org. Biomol. Chem. 2015, 13, 2464 2473

[23] aL. A. Haines, K. Rajagopal, B. Ozbas, D. A. Salick, D. J. Pochan, J. P. Schneider, JACS 2005, 127, 17025-17029; bC. Zhang, Y. Li, X. Xue, P. Chu, C. Liu, K. Yang, Y. Jiang, W.-Q. Chen, G. Zou, X.-J. Liang, Chem Commun. 2015, 51, 4168-4171.

[24] H. Chen, S. Hou, H. Ma, X. Li, Y. Tan, Sci. Rep. 2016, 6, 20722-20722.

[25] S. Hou, P. X. Ma, Chem. Mater. 2015, 27, 7627-7635.

[26] aA. Singh, B. A. Lindquist, G. K. Ong, R. B. Jadrich, A. Singh, H. Ha, C J. Ellison, T. M. Truskett, D. J. Milliron, Angew. Chem. Int. Ed. 2015, 54 14840-14844; bP. W. Schmidt, J. Appl. Crystallogr. 1991, 24, 414-435.

[27] aH. Kumari, S. E. Armitage, S. R. Kline, K. K. Damodaran, S. R Kennedy, J. L. Atwood, J. W. Steed, Soft Matter 2015, 11, 8471-8478 bJ. llavsky, P. R. Jemian, J. Appl. Crystallogr. 2009, 42, 347-353.

[28] aD. T. Bong, T. D. Clark, J. R. Granja, M. R. Ghadiri, Angew. Chem. Int. Ed. 2001, 40, 988-1011; bT. D. Clark, J. M. Buriak, K. Kobayashi, M. P. Isler, D. E. McRee, M. R. Ghadiri, JACS 1998, 120, 8949-8962.

[29] J. D. Hartgerink, J. R. Granja, R. A. Milligan, M. R. Ghadiri, J. Am. Podiatr. Med. Assoc. 1996, 118, 43-50.

[30] aS. C. Larnaudie, J. C. Brendel, I. Romero-Canelón, C. Sanchez-Cano S. Catrouillet, J. Sanchis, J. P. C. Coverdale, J.-I. Song, A Habtemariam, P. J. Sadler, K. A. Jolliffe, S. Perrier, Biomacromolecules 2018, 19, 239-247; bS. Catrouillet, J. C. Brendel, S. Larnaudie, T. Barlow, K. A. Jolliffe, S. Perrier, ACS Macro Lett. 2016, 1119-1123; cE. D. H. Mansfield, M. Hartlieb, S. Catrouillet, J. Y. Rho, S. C. Larnaudie, S. E. Rogers, J. Sanchis, J. C. Brendel, S. Perrier, Soft Matter 2018, 14, 6320-6326; dS. C. Larnaudie, J. C. Brendel, K. A. Jolliffe, S. Perrier, ACS Macro Lett. 2017, 6, 1347-1351.

[31] S. K. Agrawal, N. Sanabria-DeLong, P. R. Jemian, G. N. Tew, S. R. Bhatia, Langmuir 2007, 23, 5039-5044

[32] S. A. Raw, Tetrahedron Lett. 2009, 50, 946-948.

[33] F. Zhang, J. llavsky, G. G. Long, J. P. G. Quintana, A. J. Allen, P. R. Jemian, Metallurgical and Materials Transactions a-Physical Metallurgy and Materials Science 2010, 41A, 1151-1158.

[34] http://www.sasview.org/. 
Entry for the Table of Contents (Please choose one layout)

Layout 1:

\section{FULL PAPER}

This study reports the first example of gel formation based on the use of cyclic peptides made of alternating D- and L- amino acids, which self-assemble in solution to form long nanotubes. Herein, a library of cyclic peptides were systemically studied for their gelation properties in various solvents, uncovering key parameters driving both organogel and hydrogel formation.

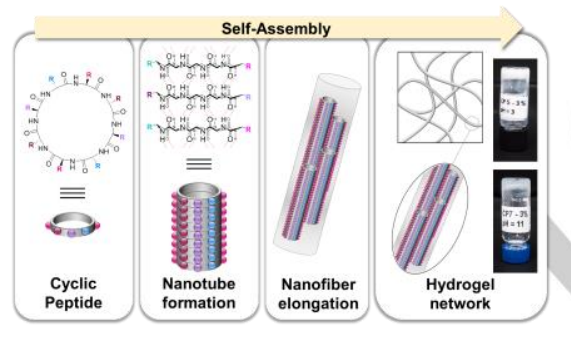

H. Shaikh, J. Y. Rho, L. J. Macdougall, P. Gurnani, A. M. Lunn, J. Yang, S. Huband, E. D. H. Mansfield, R. Peltier, ${ }^{*}$ S. Perrier*

Page No. - Page No.

Hydrogel and Organogel Formation via Hierarchical Self-Assembly of Cyclic Peptides Nanotubes 